\title{
Design and development of melt solidification of meloxicam for enhancement of solubility and dissolution
}

\author{
Anilkumar J. SHINDE 1 * (D), Rahul S. DALAVI 2 (D), Harinath N. MORE 1 (D) \\ 1 Department of Pharmaceutics, Bharati Vidyapeeth College of Pharmacy, Kolhapur, Maharashtra, India. \\ 2 Raptim Laboratories, Mumbai, Maharashtra, India \\ * Corresponding Author. E-mail: ajshinde70@rediffmail.com (A.J.S.); Tel. +91-231-263 7286.
}

Received: 08 December 2018/ Revised: 14 October 2019/ Accepted: 30 October 2019

\begin{abstract}
The objective of the present study was to prepare an amorphous system of BCS Class 2 drug meloxicam (MLX) to improve its solubility and dissolution by using the melt solidification techniques. About $40 \%$ of new chemical entities do not reach to market due to its poor aqueous solubility. Melt solidification technique is an important process to control the transition from liquid in to solid phase to obtain product in an amorphous form. During the process of heating, some solid gets melted and if quench cooled, instead of crystallizing gets converted to amorphous solid form appearing as that of glass, which improve dissolution and bioavailability of drugs. Physical mixtures of MLX were prepared by melt solidification technique using polymer (soluplus). The solubility and dissolution studies for the meloxicam and batches were conducted in a phosphate buffer ( $\mathrm{pH}$ 6.8). The fourier transform infrared(FTIR) spectrophotometry, X-ray diffraction microscopy(XDM) and Differential scanning calorimetry(DSC) studies were conducted to evaluated pure drug and optimized batch(MS7). Saturation solubility and \% drug release showed the improve solubility and dissolution, results suggest that optimized batch (MS7) containing drug and polymer in proportion of 1:4 (MLX:soluplus) was a successful enhancing the solubility and dissolution of MLX. The \% crystallinity of MLX in amorphous sample was $18.60 \%$, which indicates significant decrease in crystallinity of MLX in an amorphous system. The best fit model of optimized batch (MS7) was zero order model, showing the \%drug release $96.83 \%$ and $\mathrm{R}^{2}$ 0.9904. The present investigation, successfully enhancement solubility and dissolution of MLX by using melt solidification technique.
\end{abstract}

KEYWORDS: Meloxicam; melt solidification; dissolution; solubility; soluplus.

\section{INTRODUCTION}

Today, $30-40 \%$ of all new chemical entities (NCE) agree to from poor aqueous solubility, hence the enhancement of the solubility of the poorly water soluble drug is the one of the most challenging part of modern drug development [1-2]. Among the wide variety of parameters those delay the development of pharmaceutical products and restricts the bioavailability of oral products, solubility is the most important parameter for the formulation scientists [3-7]. Amorphous solid do not have ordered internal structure and do not melt at a definite, sharp melting point. With increase of temperature, it slowly softens, becomes less viscous and melts over a range of temperature [8-10]. They unlike from crystalline solids in that, they tend to flow, when subjected to sufficient pressure over a period of time. Amorphous solids are not solids in genuine sense; truly they are super cooled liquids. Solubility process depends on the bonding linking the solute and solvent molecule. In solubility bonds involve in solubilization are mainly dipole-dipole interactions, london forces, hydrogen bonding, ionic bonding etc [11-13]. Dissolution takes place, when the solvent is able to pull ions out of their crystal lattice or structure.

The approach for BCS Class 2 drugs, having dissolution limitations but no permeation limitation, is to increase the amount of dissolved drug molecules at the absorption site. MLX is 4-hydroxy-2-methyl-N-[(5methyl-1, 3-thiazol-2-yl)-2H-1,2-benzothiazine-3-carboxamide-1,1-dioxide. It is a COX-2 inhibitor used to treat joint diseases such as osteoarthritis, rheumatoid arthritis and other musculoskeletal disorders. It is practically insoluble in water and its solubility increases significantly with an increase in $\mathrm{pH}$. The enhancement of oral bioavailability of poorly water soluble drugs remains one of the most important aspects of drug development although salt formation, solubilization and particle size reduction have commonly

How to cite this article: Shinde AJ, Dalavi RS, More HN. Design and development of melt solidification of meloxicam for enhancement of solubility and dissolution. J Res Pharm. 2020; 24(1): 56-70. 
been used to increase the dissolution rate and thereby oral absorption and bioavailability of such drugs. The several methods employed to enhance the drug solubility such as melt solidification, freeze drying, spray drying, melting and quench cooling, melt extrusion, milling, wet granulation and desolvation have been reported to successfully prepare a disordered, high energy and unstable amorphous forms of drugs [14-17].

Melt solidification technique was used, drug goes crystalline to amorphous form, may increase the dissolution rate and solubility. It is an important process to control the transition from liquid into solid phase to obtain product in an appropriate form for their transport, storage and subsequent use. This method is an economical process, using the smallest and simplest equipment possible [18-20]. The transformation of a melt into a solid with a certain appearance and specific physical properties is an important operation in pharmaceutical industries. The solidification influence properties of the solidified melt such as shape, size, crystalline structure, and hardness. Amorphous solids are high energy states containing no long range order and presenting molecular arrangements similar to that of liquids [21,22]. Thermal glass transition $\left(\mathrm{T}_{\mathrm{g}}\right)$ is frequently used as a benchmark, when assessing the likely stability of a formulation as a function of temperature and/or other storage conditions, that can affect $T_{g}$ and it is well known that both chemical and physical instabilities of both the drug and carrier are typically more pronounced above $\mathrm{T}_{\mathrm{g}}$ compared to below $\mathrm{T}_{\mathrm{g}}[23]$.

The MLX mechanism of action, like that of other NSAIDs, may be related to prostaglandin synthetase (cyclo-oxygenase, selective COX-2 inhibitor) inhibition, which is involved in the initial steps of the arachidonic acid flow, resulting in the reduced formation of prostaglandins, thromboxanes and prostacyclin. Soluplus is a polyvinyl caprolactams polyvinyl acetate-polyethylene glycol graft co-polymer, solubiliser with an amphiphilic chemical structure, which was particularly developed for solid solutions [24,25]. Due to its bifunctional character, it is able to act as a matrix polymer for solid solutions on the one hand, and the other is capable of solubilizing poorly soluble drugs in aqueous media. The amorphous system of MLX and soluplus was prepared by the process of melt solidification technique, so as to enhance the solubility, dissolution and bioavailability in the present investigation.

\section{RESULTS AND DISCUSSION}

\subsection{Characterization of MLX}

The MLX was found to be pale yellow, odorless crystalline solid, which complies with I.P. monograph. Melting point of MLX was determined by capillary method and observed range between 251$254^{\circ} \mathrm{C}$. FTIR studies revealed that the fundamental peaks of MLX were retained in the physical mixture indicating absence of any chemical interaction between MLX and excipients used. Thus, these excipients were used in the MLX amorphous system. From the FTIR studies, it can be revealed that the principal peaks of the pure drug MLX were retained in the physical mixture was almost identical. The characteristics peak of the hydroxyl group (OH stretching) at $3282.84 \mathrm{~cm}^{-1}$, a band peak at $3327.21 \mathrm{~cm}^{-1}$ owing to amino group (N-H stretching), the characteristics peak of the carbonyl group ( $\mathrm{C}=\mathrm{O}$ stretching) present in the aldehyde at $1730.15 \mathrm{~cm}^{-1}$, a stretching $(\mathrm{S}=\mathrm{O})$ sulfur at $1049.33 \mathrm{~cm}^{-1}$ shows in Table 1. An overlain spectrum of pure MLX and physical mixture is shown in Figure 1.

Table 1. FTIR of pure MLX and physical mixture.

\begin{tabular}{cccl}
\hline $\begin{array}{c}\text { Observed peak } \\
\text { (pure MLX) }\end{array}$ & $\begin{array}{c}\text { Observed peak } \\
\text { (Physical mixture) }\end{array}$ & Reported peak & Types of peak \\
\hline 1725.24 & 1730.15 & $1720-1740$ & $\mathrm{C}=\mathrm{O}$ (aldehyde) \\
1049.33 & 1089.54 & $1000-1100$ & $\mathrm{~S}=\mathrm{O}$ Stretching \\
2888.47 & 2860.43 & $2850-3000$ & $\mathrm{CH}_{3}$ Stretching (alkanes) \\
2978.09 & 2924.09 & $2850-3000$ & $\mathrm{CH}_{2}$ Stretching \\
3282.84 & 3284.77 & $3200-3550$ & $\mathrm{OH}$ Stretching (phenol) \\
3327.21 & 3458.37 & $3400-3500$ & $\mathrm{NH}$ Weak (amines) \\
\hline
\end{tabular}




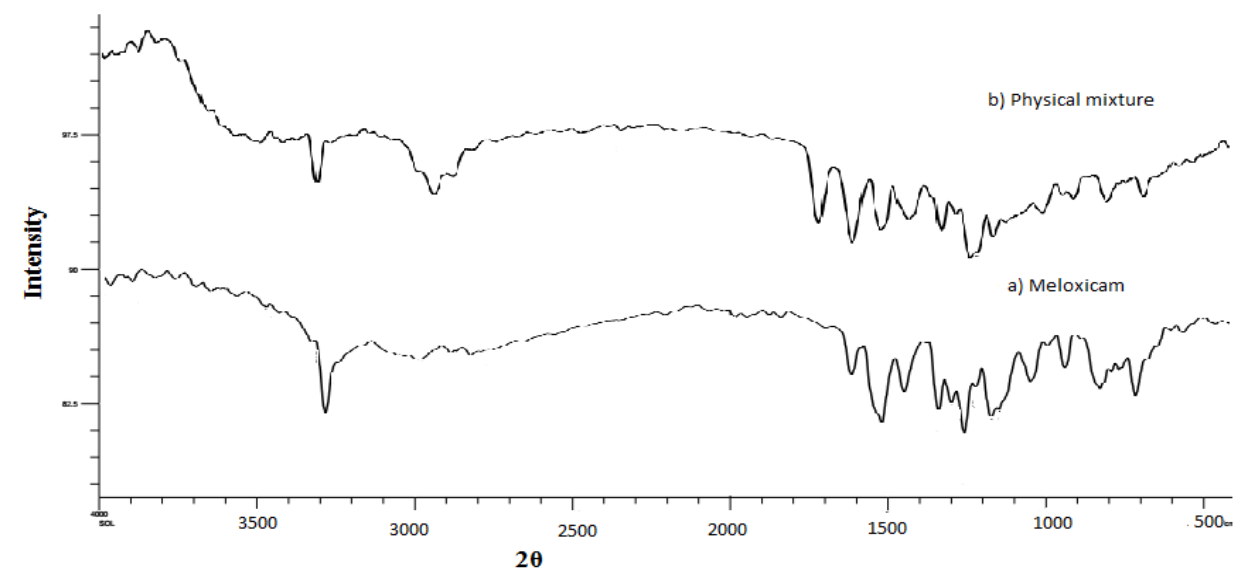

Figure 1. Overlain spectrum of a) MLX and b) physical mixture.

\subsubsection{Calibration curve in distilled water}

The calibration curve of pure MLX was found to be linear in the concentration range of $10-50 \mu \mathrm{g} / \mathrm{mL}$ at $362 \mathrm{~nm}$. i.e. the drug obeys Beer-Lambert's law in the range of 10-50 $\mathrm{gg} / \mathrm{mL}$. The absorbance values at different concentration obtained by UV double beam spectrophotometer (Jasco V630) are shown in Table 2. Using absorbance and concentration data the calibration curve was plotted as shown in Figure 2.

Table 2. Absorbance data of MLX in distilled water.

\begin{tabular}{ccc}
\hline Sr. No. & Concentration in $\mathbf{\mu g} / \mathbf{m L}$ & Absorbance at $\mathbf{3 6 2} \mathbf{~ n m}$ \\
\hline 1 & 0 & 0 \\
2 & 10 & 0.21 \\
3 & 20 & 0.41 \\
4 & 30 & 0.63 \\
5 & 40 & 0.87 \\
6 & 50 & 1.09 \\
\hline
\end{tabular}

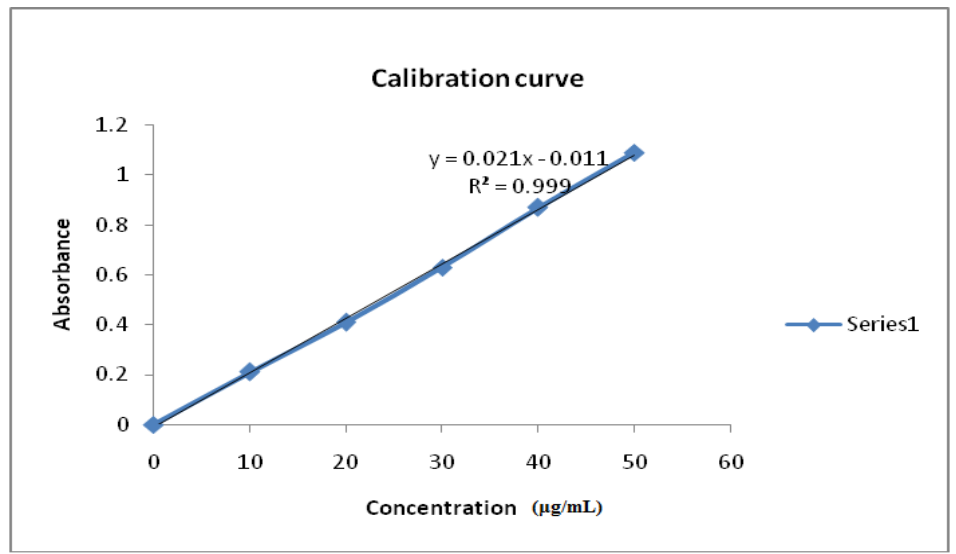

Figure 2. Calibration curve of MLX in distilled water at $362 \mathrm{~nm}$.

\subsubsection{Calibration curve in phosphate buffer $p H 6.8$}

The calibration curve of pure MLX in phosphate buffer $\mathrm{pH} 6.8$ was found to be linear in the concentration range of $10-50 \mu \mathrm{g} / \mathrm{mL}$ at $362 \mathrm{~nm}$ which shown in figure 3 . Concentration and absorbance data for calibration curve was shown in Table 3. Calibration data of MLX in phosphate buffer pH 6.8 is shown in Table 4. 
Table 3. Absorbance data for MLX in phosphate buffer $\mathrm{pH} 6.8$.

\begin{tabular}{ccc}
\hline Sr. No. & Concentration $(\boldsymbol{\mu g} / \mathbf{m L})$ & Absorbance at $\mathbf{2 6 0} \mathbf{~ n m}$ \\
\hline 1 & 0 & 0 \\
2 & 10 & 0.33 \\
3 & 20 & 0.754 \\
4 & 30 & 1.104 \\
5 & 40 & 1.421 \\
6 & 50 & 1.632 \\
\hline
\end{tabular}

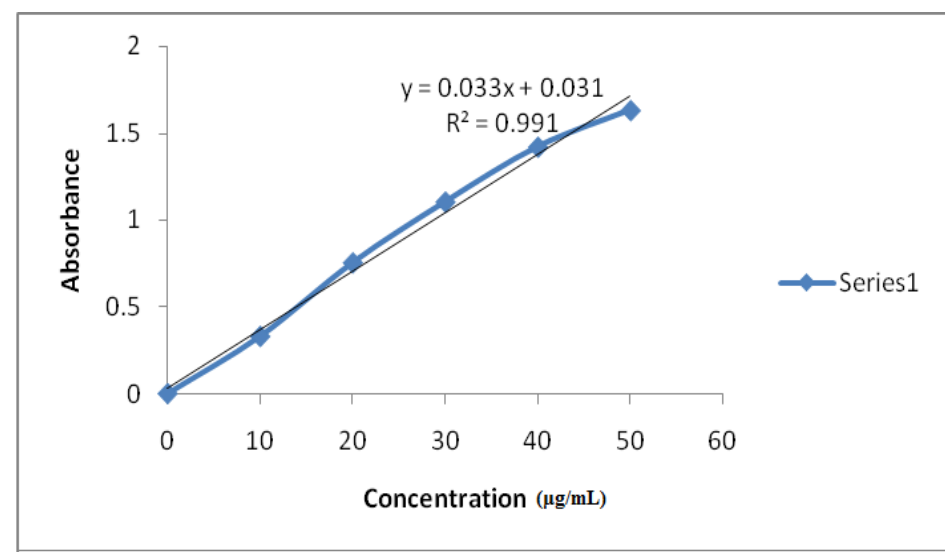

Figure 3. Calibration curve of MLX in phosphate buffer $\mathrm{pH} 6.8$.

Table 4. Calibration data of MLX in phosphate buffer $\mathrm{pH} 6.8$.

\begin{tabular}{cc}
\hline Parameters & Values \\
\hline Slope $(\mathrm{m})$ & 0.033666 \\
Intercept $(\mathrm{c})$ & 0.031857 \\
Correlation & 0.991 \\
\hline
\end{tabular}

\subsubsection{Solubility of MLX}

The results of the solubility study reveals that pure MLX has shown highest solubility in phosphate buffer pH 6.8 as compare to distilled water shown in Table 5. The solubility of MLX showed $0.025 \mathrm{mg} / \mathrm{mL} \&$ $0.040 \mathrm{mg} / \mathrm{ml}$ in distilled water and phosphate buffer $\mathrm{pH} 6.8$ respectively.

Table 5. Saturation solubility of MLX in various solvents.

\begin{tabular}{ccc}
\hline Solvents & Drug & Solubility $(\mathrm{mg} / \mathrm{mL})$ \\
\hline Distilled water & Pure drug & 0.025 \\
Phosphate buffer pH6.8 & Pure drug & 0.040 \\
\hline
\end{tabular}

\subsection{UV absorption spectra}

Solution of MLX was prepared in phosphate buffer pH 6.8 and scanned from $200 \mathrm{~nm}$ to $400 \mathrm{~nm}$ wavelength using UV visible spectrophotometer found to be $362 \mathrm{~nm}$ shown in Figure 4. 


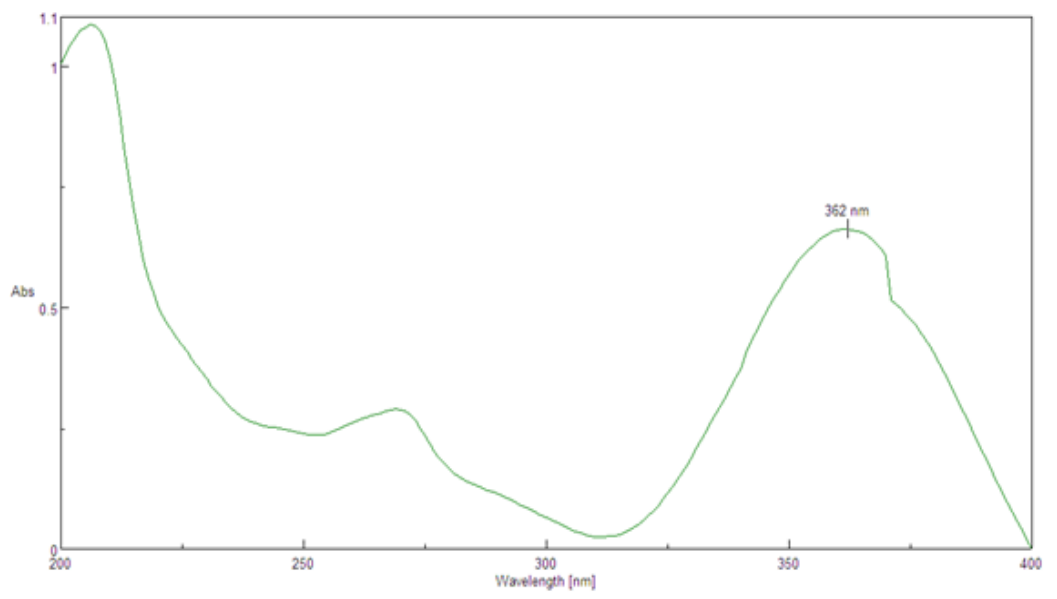

Figure 4. UV spectra of MLX.

\subsection{Formulation of MLX amorphous system}

\subsubsection{Selection of MLX and soluplus proportions for trial batches}

Evaluate the various concentrations of MLX and soluplus for different ratios. The ratio was used 1:0.25, 1:0.50, 1:0.75, 1:1, 1:1.25, 1:1.50 (MLX:soluplus) results observe less solubility of amorphous system. As per literature and as the ratio of 1:1, 1:0.50, 1:2, 1:2.5, 1:3, 1:3.5, 1:4 was used as further development of formulation. The solubility results of MLX amorphous system $0.252 \mathrm{mg} / \mathrm{mL}$ were shown in Table 6 .

Table 6. Selection of MLX and soluplus proportions.

\begin{tabular}{ccc}
\hline Sr. No. & Ratios & Solubility $(\mathrm{mg} / \mathrm{mL})$ \\
\hline 1 & $1: 0.25$ & 0.112 \\
2 & $1: 0.50$ & 0.168 \\
3 & $1: 0.75$ & 0.208 \\
4 & $1: 1$ & 0.241 \\
5 & $1: 1.25$ & 0.243 \\
6 & $1: 1.50$ & 0.252 \\
\hline
\end{tabular}

\subsection{Preparation of MLX amorphous system}

The MLX amorphous system was prepared by using soluplus in proportions of 1:1, 1:1.5, 1:2, 1:2.5, 1:3, 1:3.5, 1:4 (MLX:soluplus). The drug and polymer were triturated in dried mortar for $5 \mathrm{~min}$ and this mixture was melted, converted aqueous phase, then quench cooling to converted glassy state by trituration to amorphous form. Preparations ratios as shown in Table 7.

Table 7. Preparation of MLX and soluplus proportions.

\begin{tabular}{cccc}
\hline Batches & Meloxicam $(\mathbf{m g})$ & Soluplus $(\mathbf{m g})$ & Ratios \\
\hline MS 1 & 50 & 50 & $1: 1$ \\
MS 2 & 50 & 75 & $1: 1.5$ \\
MS 3 & 50 & 100 & $1: 2$ \\
MS 4 & 50 & 125 & $1: 2.5$ \\
MS 5 & 50 & 150 & $1: 3$ \\
MS 6 & 50 & 175 & $1: 3.5$ \\
MS 7 & 50 & 200 & $1: 4$ \\
\hline
\end{tabular}




\subsection{Characterization of MLX amorphous system}

\subsubsection{Saturation solubility of MLX amorphous system}

The optimized batch MS7 showed highest solubility in water $(0.451 \mathrm{mg} / \mathrm{mL})$, as compared with pure $\operatorname{MLX}(0.025 \mathrm{mg} / \mathrm{mL})$, which was 18 -fold greater than pure MLX respectively and formulation of amorphous system has also shown to enhance the solubility in water. Thus, solubility of formulations was improved 18.56 fold compared to pure MLX. The improvement in solubility of MLX amorphous system is shown in Table 8.

Table 8. Solubility of pure MLX and prepared batches

\begin{tabular}{ccc}
\hline Batches & Ratio & Solubility $(\mathbf{m g} / \mathbf{m L})$ \\
\hline MLX & Pure drug & 0.025 \\
MS 1 & MS 1 & 0.253 \\
MS 2 & MS 2 & 0.258 \\
MS 3 & MS 3 & 0.279 \\
MS 4 & MS 4 & 0.279 \\
MS 5 & MS 5 & 0.367 \\
MS 6 & MS 6 & 0.402 \\
MS 7 & MS 7 & 0.451 \\
\hline
\end{tabular}

\subsection{In vitro dissolution studies}

In vitro release profile of all MLX amorphous system formulations in phosphate buffer $\mathrm{pH} 6.8$ were shown in Figure 5. MLX amorphous system was showed significantly higher \% drug release as compared with all batches of formulation. The cumulative percentage release of optimized batch MS7 was observed 96.83\%. The interaction between MLX and soluplus for the optimized batch MS 7, the soluplus ratio increase as compare with pure MLX results for an enhancement of dissolution. The best fit model of optimized batch (MS 7) was zero order model, showing the \%drug release $96.83 \%$ and $\mathrm{R}^{2} 0.9904$ shown in Table 9.

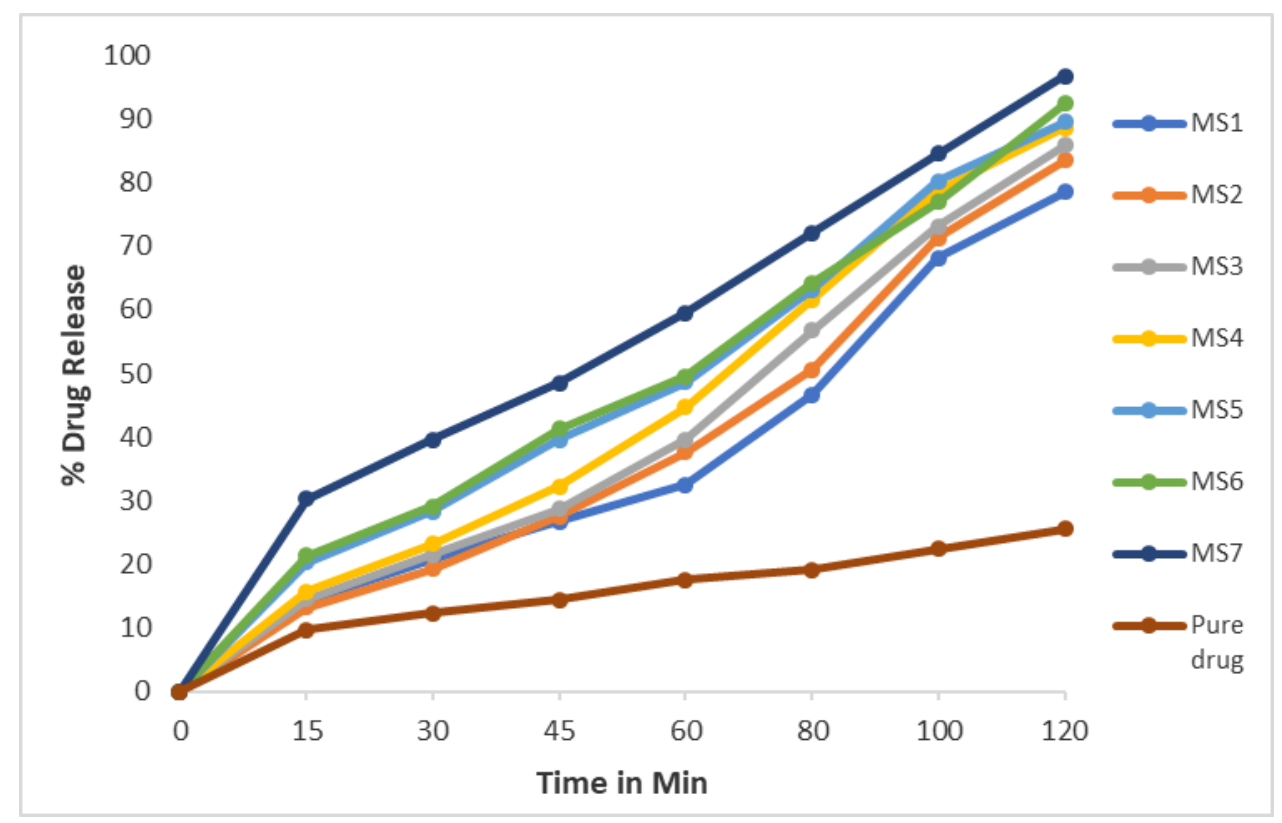

Figure 5. In vitro release profile of formulation MS1 to MS7 and pure drug. 
Table 9. Model fitting of batches MS1- MS7.

\begin{tabular}{clccc}
\hline Batch code & Model fit & $\mathbf{R}^{\mathbf{2}}$ & $\mathbf{n}$ & Constant \\
\hline MS 1 & Zero order & 0.9894 & 0.8721 & 1.2321 \\
MS 2 & Zero order & 0.9812 & 0.8545 & 1.2012 \\
MS 3 & Zero order & 0.9865 & 0.8632 & 1.322 \\
MS 4 & Zero order & 0.9882 & 0.8433 & 1.4131 \\
MS 5 & Zero order & 0.9832 & 0.7831 & 1.3312 \\
MS 6 & Zero order & 0.9901 & 0.8594 & 1.4042 \\
MS 7 & Zero order & 0.9931 & 0.7855 & 1.0412 \\
\hline
\end{tabular}

\subsection{Solid state characterization studies}

2.7.1. FTIR spectrophotometry studies for amorphous system

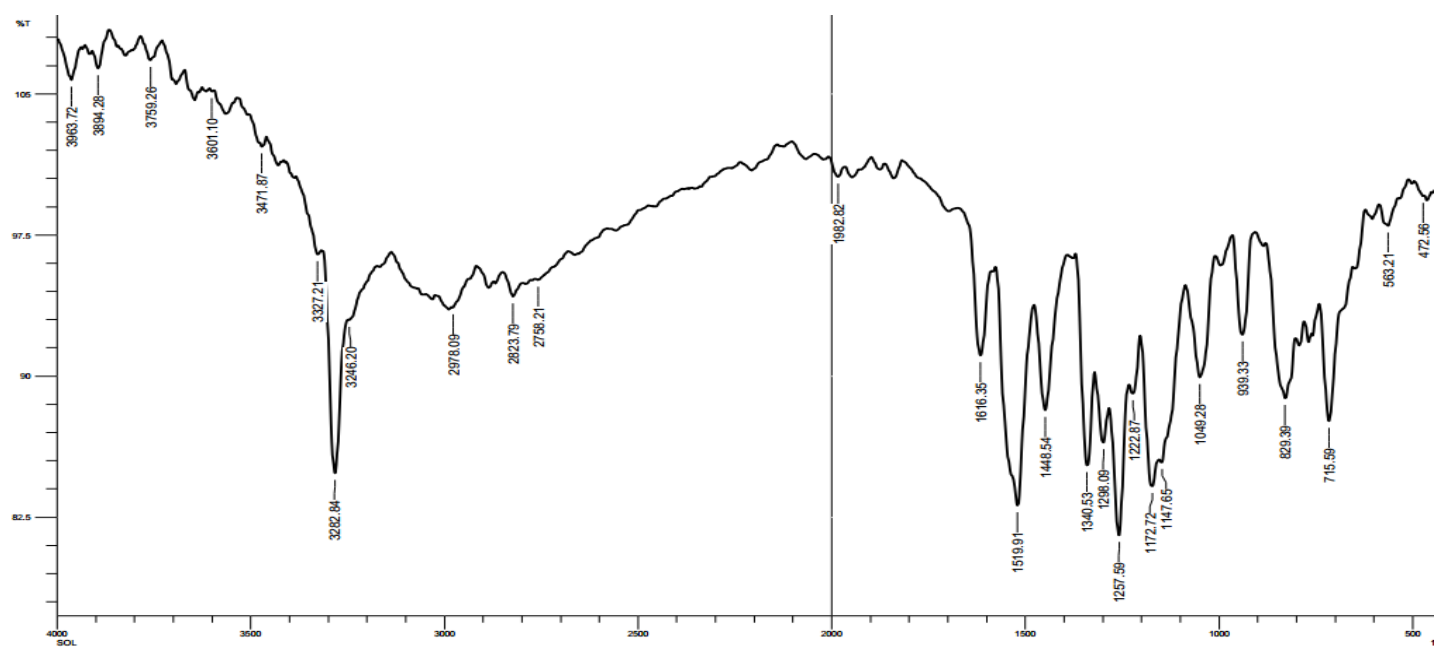

Figure 6. FTIR of MLX.

Table 10. FTIR peak of MLX.

\begin{tabular}{ccl}
\hline Observed peak $\left.\mathbf{( c m}^{-1}\right)$ & Reported peak $\left(\mathbf{c m}^{-1}\right)$ & Types of peak \\
\hline 3282.84 & $3200-3550$ & O-H stretching (phenol) \\
2978.09 & $2850-3000$ & $\mathrm{CH}_{3}$ stretching \\
3327.21 & $3300-3400$ & $\mathrm{~N}-\mathrm{H}$ bend (Amine band) \\
1049.33 & $1030-1060$ & $\mathrm{~S}=\mathrm{O}$ stretching (Sulfur) \\
\hline
\end{tabular}

Peak observed at $3327.21 \mathrm{~cm}^{-1}$ was attributed to N-H stretching. Peaks at $1049.33 \mathrm{~cm}^{-1}$ was attributed to presence of sulfur band, $3282.84 \mathrm{~cm}^{-1}$ attributes to O-H (phenol) stretching, peaks at $2978.09 \mathrm{~cm}^{-1}$ attributed to $\mathrm{C}-\mathrm{H}$ stretching, $\mathrm{O}-\mathrm{H}$ stretching and $\mathrm{S}=\mathrm{O}$ amide stretching respectively as shown in Figure 6 and Table 10. As the peak intensity, OH(phenol) of MLX is more intense, when compared to optimized batch formulation. As a result, hydrogen bonding between drug and optimized batch MS 7 showing hydrophilicity increases as shown in Figure 7 and Table 11. 


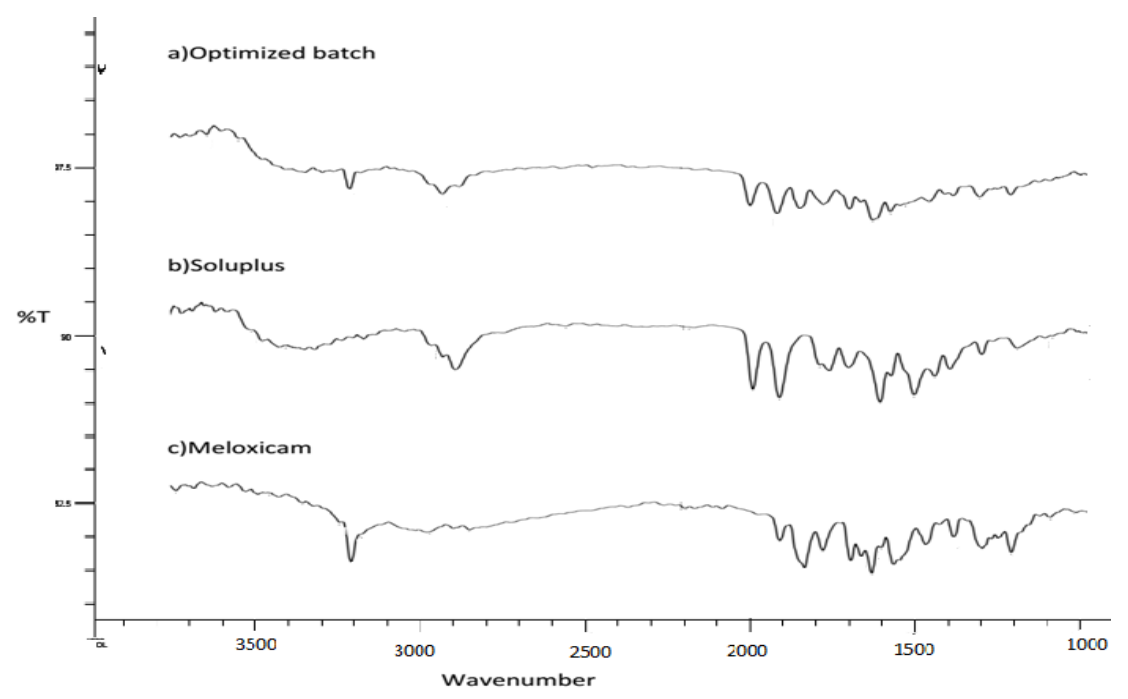

Figure 7. Overlain of FTIR spectra of a) optimized batch, b) soluplus c) MLX.

Table 11. Overlain FTIR spectra of pure MLX and optimized batch.

\begin{tabular}{cccl}
\hline $\begin{array}{c}\text { Observed peak } \\
\text { (pure MLX) } \\
\left(\mathbf{c m}^{-1}\right)\end{array}$ & $\begin{array}{c}\text { Observed peak } \\
\text { (optimized batch) } \\
\left(\mathbf{c m}^{-1}\right)\end{array}$ & $\begin{array}{c}\text { Reported peak } \\
\left(\mathbf{c m}^{-1}\right)\end{array}$ & Types of peak \\
\hline 1725.24 & 1730.15 & $1720-1740$ & $\mathrm{C}=\mathrm{O}$ (aldehyde) \\
2888.47 & 2860.43 & $2850-3000$ & $\mathrm{CH}_{3}$ Stretching (alkanes) \\
2978.09 & 2924.09 & $2850-3000$ & $\mathrm{CH}_{2}$ Stretching \\
3282.84 & 3284.77 & $3200-3550$ & $\mathrm{OH}$ Stretching (phenol) \\
3327.21 & 3458.37 & $3400-3500$ & NH weak (amines) \\
\hline
\end{tabular}

2.7.2. Differential scanning calorimetry studies

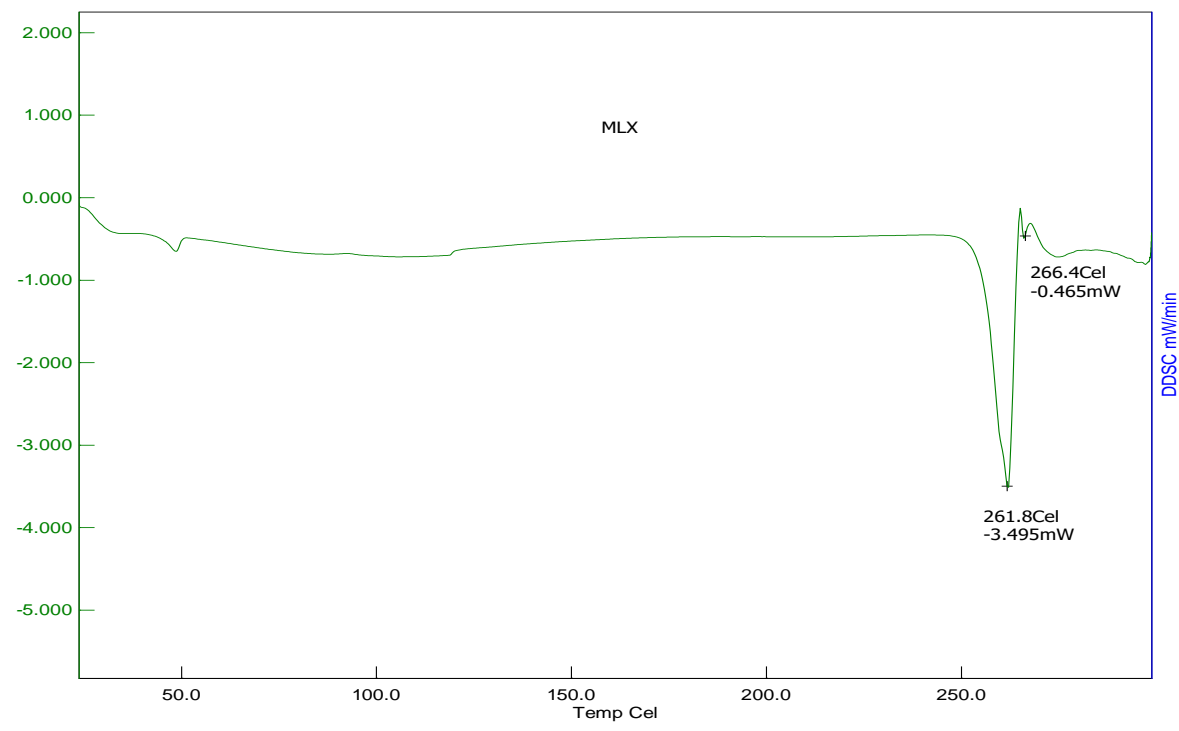

Figure 8. DSC of MLX.

The DSC of MLX was showing a sharp endothermic peak at $261.8^{\circ} \mathrm{C}$, which indicates its melting point and the sharp peak indicates its crystalline nature. 
DSC can be used to investigate and predict the changes in crystalline form of the drug. Figure 8 \& 9 shows the results of the MLX pure drug and the formulation (MS7). The pure drug exhibited a large and sharp endothermic peak at $261.8^{\circ} \mathrm{C}$ indicating the near to melting point. DSC thermogram of optimized batch (MS7) showed an endothermic peak at $191.9^{\circ} \mathrm{C}$ described shifting the peak as compared MLX. However, the endothermic peak of MLX shifted about the left due to reduction of the crystal sizes. Besides this, no additional peaks were found to demonstrate the significant changes in the melting characteristics of MLX in the formulation indicating no polymorphic changes in the MLX. The peaks were found to be nearly identical, with a calculated enthalpy $(\Delta \mathrm{H})$ of pure drug and optimized batch were $122 \mathrm{~J} / \mathrm{g}$, and $22.8 \mathrm{~J} / \mathrm{g}$ respectively.

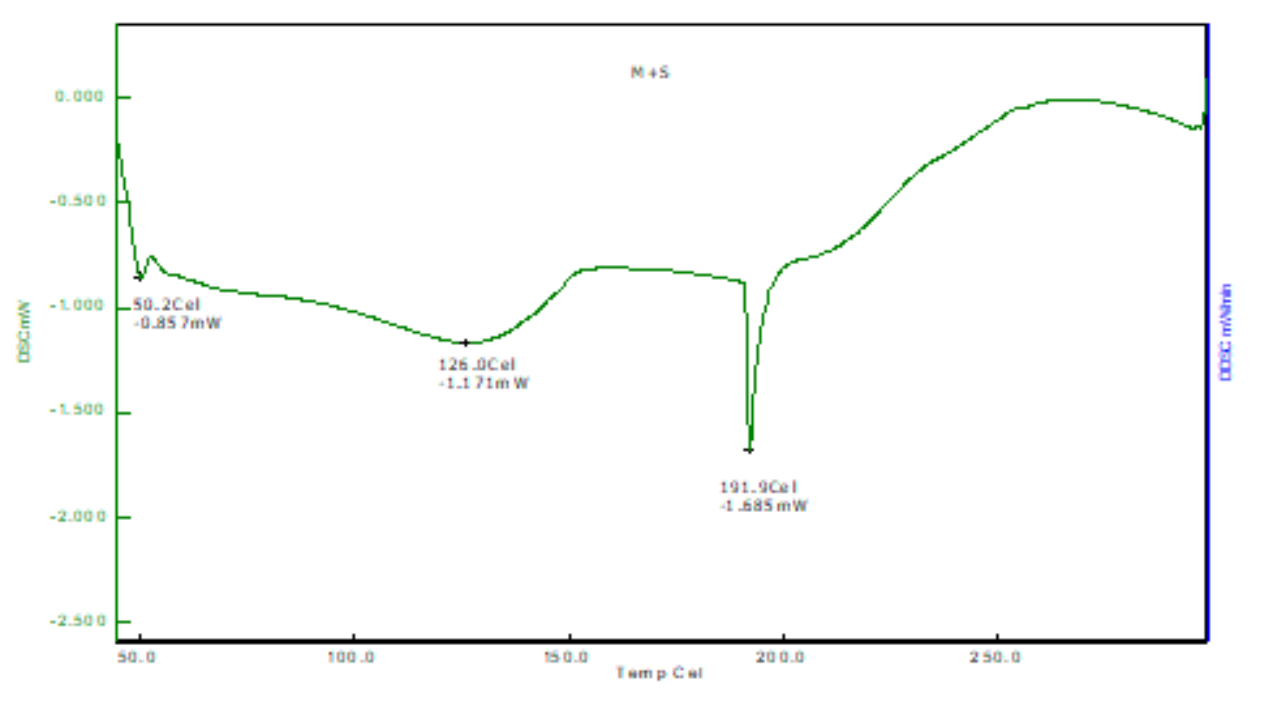

Figure 9. DSC of optimized batch (MS7).

\subsubsection{Powder $x$-ray diffraction microscopy studies}

PXDM studies results of MLX shows that intense peaks at 15.5, 22.1, 22.5 degree theta. The intensity of these peaks were high, thus MLX was found to be crystalline in nature as shown in Figure 10.

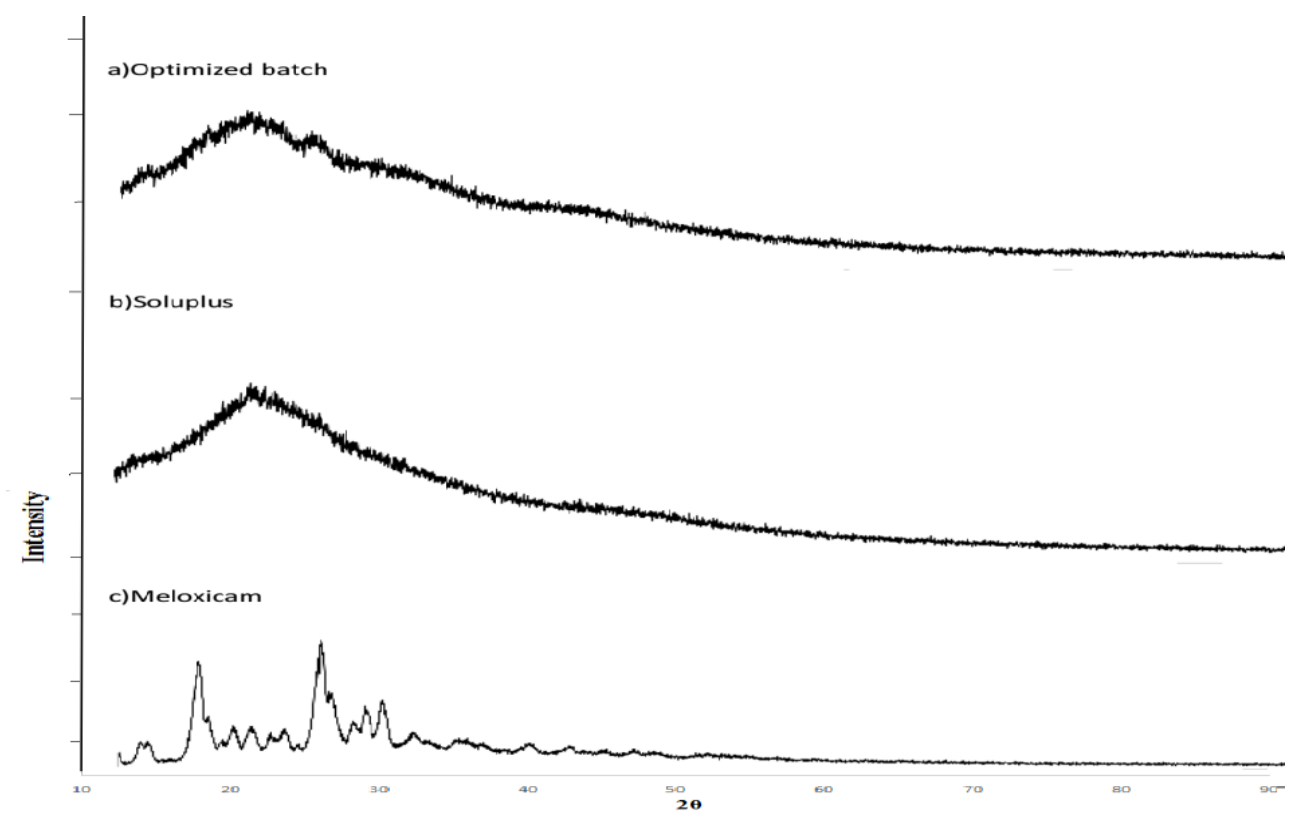

Figure 10. Overlain of XRD of amorphous system a) optimized batch, b) soluplus, c) MLX. 
Amorphous system of (optimized batch MS7) showed peaks at 13.10, 14.5, 19.5, 21.9, 22.7, 24.21 with less intensity as compare to pure MLX. This indicates that MLX crystallinity was decreased.

\subsubsection{Glass transition temperature $\left(T_{g}\right)$}

The glass transition temperature of amorphous samples was predicted theoretically using the GordonTaylor equation (1) \& (2):

$$
\begin{aligned}
& \operatorname{Tg} \text { mix }=\frac{W 1 \mathrm{Tg} 1+\mathrm{kw} 2 \mathrm{Tg} 2}{w 1+k w 2} \times 100 \\
& \mathbf{k}=\frac{\sigma 1 \mathrm{Tg} 1}{62 \mathrm{Tg} 2} \\
& \mathrm{~T}_{\mathrm{g} 1}=21.2^{\circ} \mathrm{C}, \mathrm{T}_{\mathrm{g} 2}=-42^{\circ} \mathrm{C} \\
& \mathrm{w}_{1}=0.615, \mathrm{w}_{2}=0.265 \\
& \mathrm{~T}_{\mathrm{g} \text { mix }}=27.56^{\circ} \mathrm{C}
\end{aligned}
$$

Theoretically the glass transition temperature of the mixture has increased significantly to such an extent, it can be concluded that the mixture will be stable at room temperature, thus improving processing properties of both the drugs.

\subsection{5. \% Crystallinity study}

The percentage of crystalline material, present in the samples were calculated, by using equation (3).

$$
\begin{aligned}
& \text { \%Crystallinity }=\frac{\Delta H(\text { amorphous sample })}{\Delta H 100} \times 100 \\
& \text { \%Crystallinity }=\frac{\Delta H(22.7)}{\Delta H 100} \times 100
\end{aligned}
$$

Where, $\Delta \mathrm{H}$ is the melting enthalpy of the amorphous sample, $\Delta \mathrm{H}$ is the Enthalpy of melting of proportional $100 \%$ of pure crystalline drug.

The \% crystallinity of MLX in amorphous sample was $18.60 \%$, which indicates significant decrease in crystallinity of MLX in amorphous system as shown Table 12.

Table 12. Enthalpy and \% crystallinity of MLX, physical mixture, amorphous system.

\begin{tabular}{cccc}
\hline Parameters & Pure Drug & Physical mixture & Amorphous system \\
\hline Enthalpy(J/g) & 122 & 86.35 & 22.7 \\
\% Crystallinity & 100 & 70.83 & 18.60 \\
\hline
\end{tabular}

\subsection{Scanning electron microscopy}

Surface morphology of the formed crystals were determined by using SEM. The result reveals that the crystalline nature of all the formulations remains with slight change in crystallinity. The SEM images and particle size distributions and its morphology of the MLX amorphous system was presented in Figure 11. Amorphisms were found to be crumbling in shape with a narrow particle size distribution. 


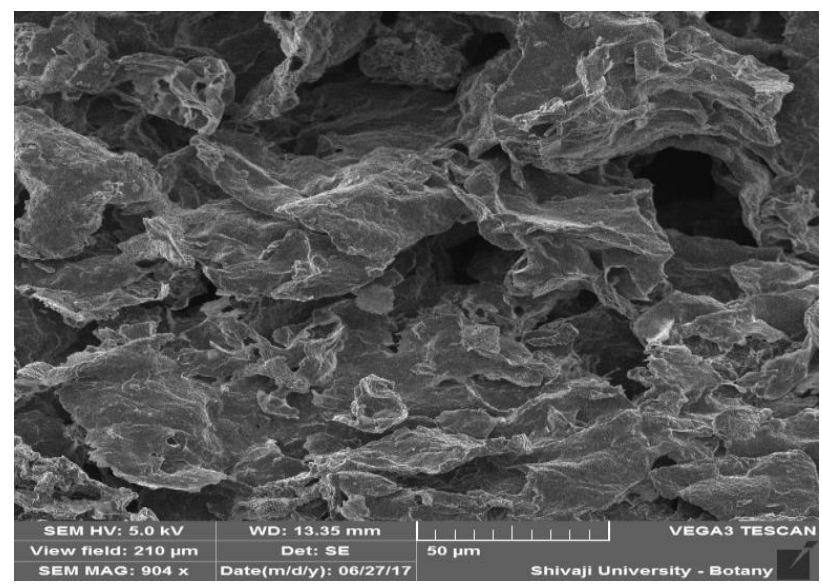

Figure 11. SEM of optimized batch (MS 7) at $5.0 \mathrm{kv} \times 50 \mu \mathrm{m}$.

\subsection{Acccelerated stability studies}

\subsubsection{In vitro dissolution studies}

In vitro dissolution studies after first to three month of stability studies in the phosphate buffer $\mathrm{pH} 6.8$ media shows very slight decrease in the drug release, thus indicating that the formulation was stable up to three months as shown in Figure 12.

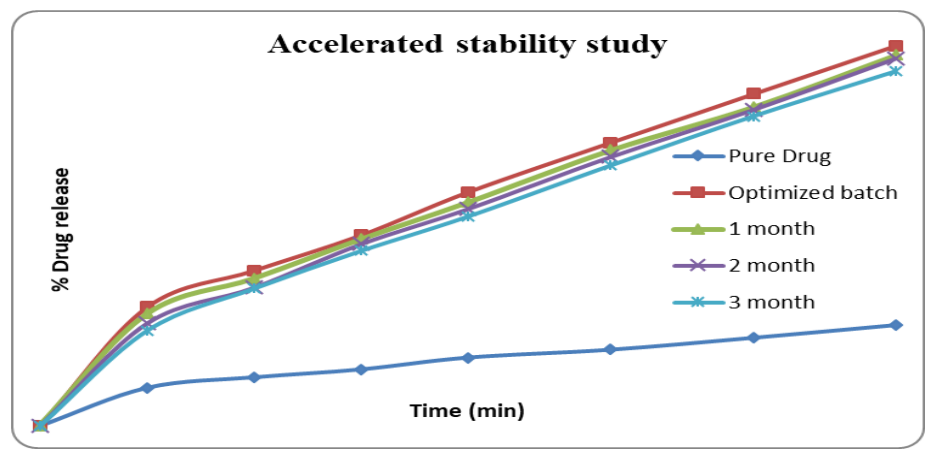

Figure 12. In vitro release profile of formulation pure drug, MS7, 1 month, 2 months, 3 months accelerated stability.

\subsubsection{Differential scanning calorimetry studies}

DSC thermogram and \% crystallinity after 3 month were shows slight increase in enthalpy and changes in the melting point. The \% crystallinity of MLX after 3 months was $16.4 \%$, which confirmed that MLX was stabilized shown in Figure 13.

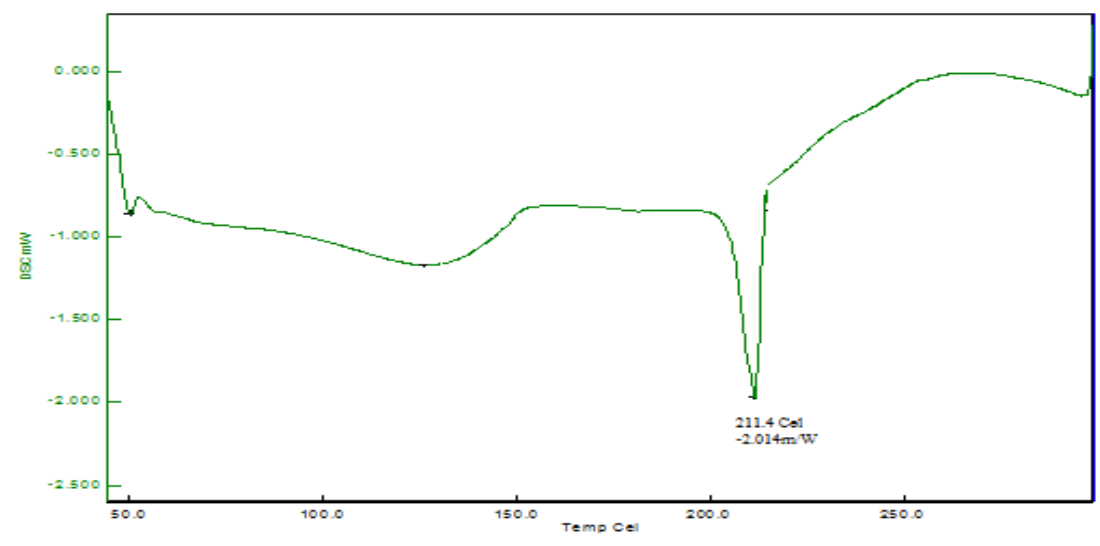

Figure 13. DSC spectra of after 3 month stability. 


\subsubsection{Powder $x$-ray diffraction microscopy studies}

PXDM studies of the MLX showed increases in intensity of the peaks. The decrease in intensity of peak in amorphous form of optimized batch after 3 month stability studies, amorphous sample as shown in Figure 14. Thus, amorphous sample was found to be stable after three months of accelerated stability studies.

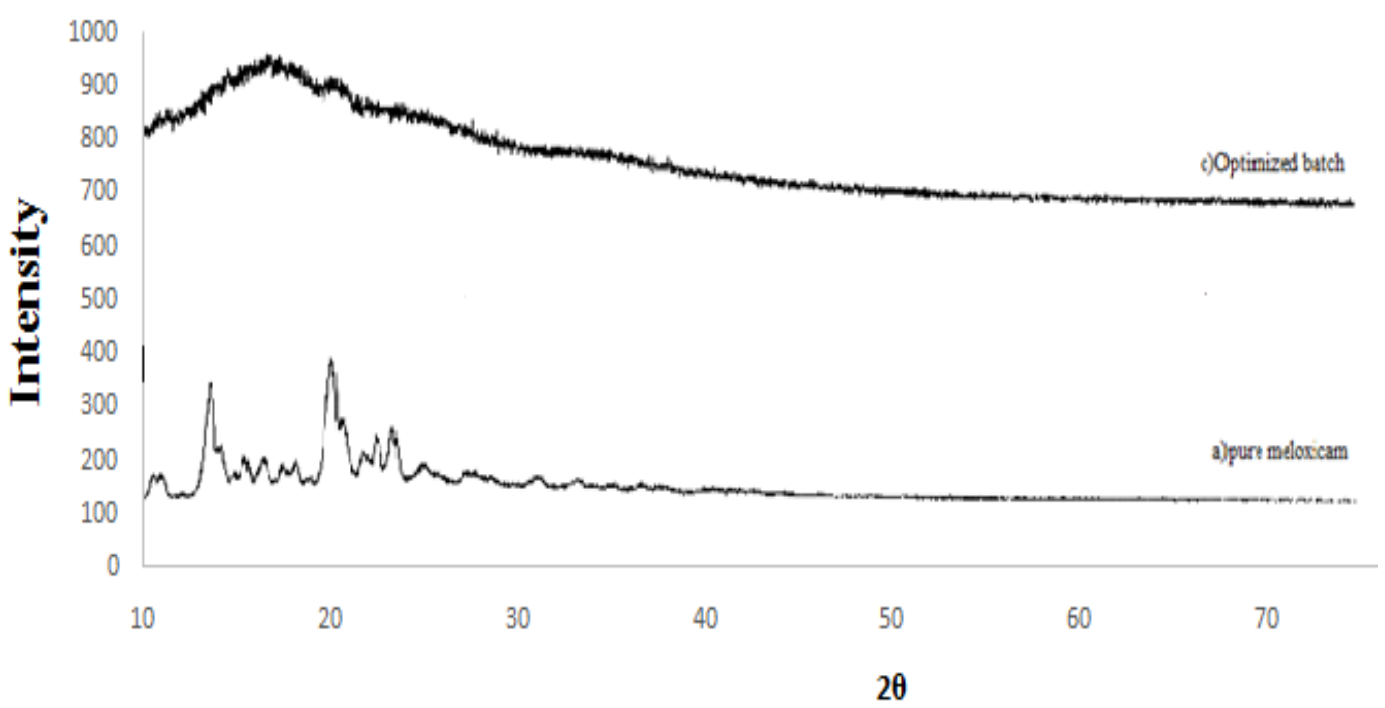

Figure 14. XRD spectra of after 3 month stability a) pure MLX, b) optimized batch (MS7).

\section{CONCLUSION}

It can be concluded that improvement in the solubility and dissolution of the water insoluble drug MLX was a successfully achieved through using melt solidification technique. The amorphous system increased solubility of MLX by 18.4 folds, \% drug release was $96.83 \%$ within 120 minutes, as compare pure MLX showed 25.54\%. FTIR-spectrophotometry studies showed that shift in the peaks of MLX. The shift during peaks were due to hydrogen bonding between drug. PXDM \& DSC studies of amorphous sample shows endotherms with less enthalpy, which indicates that drug possesses less crystallinity as compared to pure MLX and the decrease in melting point was due to molecular interaction between drug and polymer. The \% crystallinity of MLX in amorphous sample was $18.60 \%$, which indicates significant decrease in crystallinity of MLX in amorphous system. Accelerated stability studies, showed slight changes in the PXDM and DSC results of amorphous sample thus confirming stabilization of MLX.

\section{MATERIALS AND METHODS}

MLX drug was procured from Zydus Hetero drugs Ltd. Hyderabad, India, soluplus was obtained as a gift sample from BASF Corporation, Mumbai, India. All other chemicals were of analytical grade.

\subsection{Preparation of amorphous system}

The amorphous system of MLX and soluplus was prepared by the process of melt solidification technique. This technique of pure MLX and soluplus mixture of different proportion of both the drug and polymer was carried out. The MLX was prepared by using polymer (soluplus) in proportions of 1:1, 1:1.5, 1:2, 1:2.5, 1:3, 1:3.5, 1:4 (drug:polymer) as shown in table 13. The drug and polymer triturated in dried mortar for $5 \mathrm{~min}$. and mixture was melted, converted aqueous phase and quench cooling to glassy state using mortar and pestle, triturate to amorphous form of mixture $[26,27]$. 
Table 13. Preparation MLX amorphous system.

\begin{tabular}{cccc}
\hline Batches & Ratios & MLX $(\mathbf{m g})$ & Soluplus(mg) \\
\hline MS 1 & $1: 1$ & 50 & 50 \\
MS 2 & $1: 1.5$ & 50 & 75 \\
MS 3 & $1: 2$ & 50 & 100 \\
MS 4 & $1: 2.5$ & 50 & 125 \\
MS 5 & $1: 3$ & 50 & 150 \\
MS 6 & $1: 3.5$ & 50 & 175 \\
MS 7 & $1: 4$ & 50 & 200 \\
\hline
\end{tabular}

\subsection{Evaluation of amorphous system}

\subsubsection{Saturation solubility}

Weighed amount of MLX (pure drug) 10mg and the amorphous system equivalent to $10 \mathrm{mg}$ of the drug were separately introduced into $25 \mathrm{ml}$ stoppered conical flasks containing $10 \mathrm{ml}$ distilled water, phosphate buffer $\mathrm{pH} 6.8$. The sealed flasks were agitated on a rotary shaker for $48 \mathrm{~h}$ at $37^{\circ} \mathrm{C}$ and equilibrated for 2 days. An aliquot was passed through $0.45 \mu \mathrm{m}$ membrane filter and the filtrate was suitably diluted and analyzed for drug content by using UV Spectrophotometer at 362nm wavelength.

\subsubsection{In vitro drug release studies}

In vitro dissolution kinetics of MLX was carried out by using USP Type-II dissolution test apparatus. The dissolution medium was used about $900 \mathrm{ml}$ of distilled water and phosphate buffer $\mathrm{pH} 6.8$ and temperature maintained at $37.5 \pm 0.5^{\circ} \mathrm{C}$ and speed kept constant at $100 \mathrm{rpm}$. Samples $(5 \mathrm{~mL})$ were withdrawn at predetermined time intervals, immediately replaced with fresh dissolution medium and analyzed for MLX content by using UV Spectrophotometer at $362 \mathrm{~nm}$ wavelength after suitable dilution. Percent of MLX dissolved at various time intervals was calculated from the regression equation generated from the suitably constructed calibration curve.

\subsubsection{Crystallinity and thermal studies}

FTIR, PXRD and DSC studies has been extensively used for the study of \% crystallinity, glass forming abilities- glass transition (Tg) and other thermal events at microscopic level studies of melt solidification of individual drug samples.

\subsubsection{Fourier transform infrared spectrophotometry studies}

FTIR spectrum shows the fundamental peaks corresponding to the chemical nature of the drug and excipients. FTIR spectrophotometry studies were carried out in order to determine any possible interaction among drug and excipients used. Samples prepared in $\mathrm{KBr}$ discs were subjected to FTIR recording on FTIR spectrophotometry (Jasco- V-530 model) instrument. Data were collected over a spectral range of 4000 to 400 $\mathrm{cm}^{-1}$. Spectra were recorded over the wave number $400-4000 \mathrm{~cm}^{-1}$. Infrared spectrums of pure drug and optimized batch were recorded.

\subsubsection{Powder $x$-ray diffraction microscopy studies}

This technique allows amorphous and crystalline materials to be differentiated. Crystalline materials display many diffraction bands, whereas amorphous compounds present a more or less regular baseline. PXDM patterns of individual drug samples were obtained by Philips X-ray diffractometer, PW-3710, Holland, using $\mathrm{Cu} \mathrm{Ka}$ radiation $(\lambda=1.5405 \AA)$ at voltage of $40 \mathrm{kV}$, and $30 \mathrm{~mA}$ current. The data recorded over a range of $10^{\circ}$ to $50^{\circ}$ at a scanning rate of $5 \times 103 \mathrm{cps}$ using a chart speed of $5 \mathrm{~mm} / 2^{\circ}$.

\subsubsection{Differential scanning calorimetry}

The DSC, thermal analysis measures a physical property of a substance as a function of temperature. Crystalline transitions, fusion, evaporation and sublimation were easily quantified by DSC curves. DSC thermograms of drug samples were obtained by using DSC 821e (Mettler Toledo, Switzerland) instrument, operating with STARe software, version 5.1, and equipped with an intracooler. The samples (3-10 mg) were 
heated $30^{\circ} \mathrm{C}$ to $300^{\circ} \mathrm{C}$ at the rate of $10^{\circ} \mathrm{C} / \mathrm{min}$. under dry nitrogen purge $(80 \mathrm{ml} / \mathrm{min})$ in crimped and pinholed aluminum pans. Samples were scanned at well above the respective melting points.

\subsubsection{Glass transition temperature}

The glass transition temperature of amorphous system drug samples can be predicted using the Gordon-Taylor equation (1 \& 2).

\subsubsection{Percentage crystallinity}

The percentage of crystalline material, present in the samples were calculated by using equation (3).

\subsection{Scanning electron microscopy}

Evaluation of particle size and morphology was achieved via scanning electron microscopy, using a JSM-6360 microscope (JEOL, Tokyo, Japan). Samples were scattered on a thin film of a two-component epoxy resin and then coated with a platinum layer. Acceleration during the observation was $25 \mathrm{kV}$.

\subsection{Stability studies}

Stability studies were carried out according to ICH guidelines Q1A (R2). The stability of MLX amorphous system on optimized formulation MS7 were studied at $5^{\circ} \pm 3^{\circ} \mathrm{C}$ and $25^{\circ} \pm 2^{\circ} \mathrm{C}$ and $60 \% \pm 5 \% \mathrm{RH}$ for 3 months. Periodically, samples were withdrawn and the particle size as well as drug content was determined. They were withdrawn at specified intervals for analysis over a period of 30 days, 60 days and 90 day.

Acknowledgements: Anilkumar J. Shinde and other co-authors are wished to acknowledge Zydus Hetero drugs Ltd. Hyderabad, India, and BASF Corporation, Mumbai, India for providing meloxicam \& soluplus as gift samples. Authors are also grateful to Dr. H. N. More, Principal Bharati Vidyapeeth College of Pharmacy, Kolhapur for providing excellent facility to carry out this work.

Author contributions: Concept - A.S., R.D., H.M.; Design - A.S., R.D., H.M.; Supervision - A.S., H.M.; Materials A.S., R.D.; Data Collection and/or Processing - A.S., R.D.; Analysis and/or Interpretation - A.S., R.D., H.M.; Literature Search - A.S., R.D.; Writing - A.S.; Critical Reviews - A.S., R.D., H.M.

Conflict of interest statement: We wish to confirm that there are no known conflicts of interest associated with this publication and there has been no significant financial support for this work that could have influenced its outcome.

\section{REFERENCES}

[1] Reppas C, Kalantzi L. Biowaiver monographs for immediate release solid oral dosage forms: Acetaminophen. J Pharm Sci. 2006; 95(1): 4-14. [CrossRef]

[2] Alleso M, Chieng N, Rehder S, Rantanen J, Rades T, Aaltonen J. Enhanced dissolution rate and synchronized Release of drugs in binary systems through formulation: Amorphous naproxen-cimetidine mixtures prepared by mechanical activation. J Control Rel. 2009; 136: 45-53. [CrossRef]

[3] Hancock B, Zografi G. Characteristics and significance of the amorphous state in pharmaceutical systems. J Pharm Sci.1997; 86: 1-12. [Cross Ref]

[4] Kaushal A, Bansal K. Thermodynamic behavior of glassy state of structurally related compounds. Eur J Pharm Biopharm. 2008; 69: 1067-1076. [CrossRef]

[5] Bodmeier R, Wang J, Bhagwatwar H. Process and formulation variables in the preparation of wax microparticles by melt dispersion technique for water insoluble drugs. J Microcapsulation.1992; 9: 89-98. [CrossRef]

[6] Ambike A, Mahadik K, Paradkar A. Stability study of amorphous valdecoxib. Int J Pharm.2004; 282: 151-162. [CrossRef]

[7] Shinde R, Shelake M, Shetty S, Chavan Patil A, Pore Y, Late S. Enhanced solubility and dissolution rate of lamotrigine by inclusion complexation and solid dispersion technique. J Pharm Pharmacol. 2008; 60(9): 1121-1129. [CrossRef]

[8] Baird J. Evaluation of amorphous solid dispersion properties using thermal analysis techniques. Adv Drug Deli Rev. 2012; 64: 396-421. [CrossRef] 
[9] Sanghvi R, Evans D, Yalkowsky S. Stacking complexation by nicotinamide: A useful way of enhancing drug solubility. Int J Pharm. 2007; 336(1): 35-41. [CrossRef]

[10] Carstensen J. Advanced pharmaceutical solids. Marcel Dekker, New York, 2001; 117-227.

[11] Hecq J, Deleers M, Fanara D, Vranckx H, Amighi K. Preparation and characterization of nanocrystals for solubility and dissolution rate enhancement of nifedipine. Int J Pharm. 2005; 299(1-2): 167-177. [CrossRef]

[12] Balania P, Wonga S. Influence of polymer content on stabilizing milled amorphous salbutamol sulphate. Int J Pharm. 2010; 391: 125-136. [CrossRef]

[13] Amina M, Myasar M, khayrallah A. Preparation and evaluation of meloxicam solid dispersion by solvent evaporation method. Int Res J Pharm. 2014; 5: 838-845.

[14] Bhende S, Jadhav N. Moringa coagulant as a stabilizer for amorphous solid AAPS PharmSciTech.2012; 13(2): 400410. [CrossRef]

[15] Amidon G, Lennernas H, Shah V, Crison J. A theoretical base for a biopharmaceutical drug classification: the correlation of in vitro drug product dissolution and in vivo bioavailability. Pharm Res. 1995; 12(3): 413-420. [CrossRef]

[16] Branham M, Moyo T, Govender T. Preparation and solid-state characterization of ball milled saquinavir mesylate for solubility enhancement. Eur J Pharma and Biopharm. 2012; 80: 194-202. [CrossRef]

[17] Chieng N, Aaltonen J, Saville D, Rades T. Physical characterization and stability of amorphous indomethacin and ranitidine hydrochloride binary systems prepared by mechanical activation. Eur J Pharm Biopharm.2009; 71: 47-54. [CrossRef]

[18] Hancock B, Zografi G. Characteristics and significance of the amorphous state in pharmaceutical systems. Pharm Sci.1997; 86: 1-12. [CrossRef]

[19] Hassan M, Najib N, Suleiman M. Characterization of glibenclamide glassy state. Int J Pharm.1991; 67(2):131-137. [CrossRef]

[20] Hilden L, Morris K. Physics of amorphous solids. J Pharm Sci. 2004; 93: 3-12. [CrossRef]

[21] Jacob S, Nair A, Patil N, Panda P. Solid state crystallinity, amorphous state, and it implications in the pharmaceutical process. Int J Pharm Sci Res. 2011; 2(3): 472-482.

[22] Karmwar P, Graeser K, Gordon K, Strachan C, Rades T. Investigation of properties and recrystallization behavior of amorphous indomethacin samples prepared by different methods. Int J Pharm.2011; 417(1-2):94-100. [CrossRef]

[23] Jadhav N, Gaikwad V, Nair K. Glass transition temperature: Basics and application in pharmaceutical sector. Asian J Pharm. 2010; 3: 83-88.

[24] Kulkarni P, Dixit, Kumar S, Johri A. Preparation and evaluation of ketoprofen beads by melt solidification technique. Pelagia Res Lib. 2010; 1(2): 31-43.

[25] Paradkar A, Maheshwari M, Ketkar A. Chouhan, B. Preparation and evaluation of ketoprofen beads by melt solidification technique. Int J Pharm. 2003; 33-42.

[26] Pokharkar V, Mandpe L, Padamwar M, Ambike A, Mahadik K, Paradkar A. Development, characterization and stabilization of amorphous form of a low Tg drug. Powder Techno. 2006; 167: 20-25.

[27] Paradkar A, Kamble R, Kadam S. Melt solidification technique: Incorporation of higher wax content in ibuprofen beads. AAPS Pharmtech Sci. 2004; 4: 325-329. [CrossRef] 